\title{
Regularity of solutions to one-dimensional and multi-dimensional problems in the calculus of variations
}

\author{
Francis Clarke \\ Institut universitaire de France et Université de Lyon \\ Institut Camille Jordan UMR 5208 \\ Université Claude Bernard Lyon 1 \\ La Doua, 69622 Villeurbanne, France \\ E-mail: clarke@math.univ-lyon1.fr
}

\begin{abstract}
We review the long-standing issue of regularity of solutions to the basic problem in the calculus of variations, in both the one-dimensional and the multidimensional settings. It is shown how certain recent results fit in with the classical ones, in particular the theories of De Giorgi and Hilbert-Haar.

Keywords: Calculus of variations; regularity; necessary conditions; existence.
\end{abstract}

\section{Introduction}

We begin in the middle, with two of the celebrated problems proposed by Hilbert in Paris in 1900:

The 20th problem: Is it not the case that every regular variational problem has a solution, provided certain assumptions on the boundary conditions are satisfied, and provided also, if need be, that the concept of solution is suitably extended?

The 19th problem: Are the solutions of regular problems in the calculus of variations always analytic?

These questions bear upon the following basic problem in the calculus of variations: to minimize the functional

$$
J(u):=\int_{\Omega} F(x, u(x), D u(x)) d x
$$

over the functions $u: \Omega \rightarrow \mathbb{R}$ assuming prescribed values on $\Gamma:=\partial \Omega$ :

$$
u(x)=\phi(x), x \in \Gamma \text {. }
$$


Here $\Omega$ is a domain in $\mathbb{R}^{n}$ : an open bounded connected set, and $D u$ denotes the gradient of $u$. In fact, Hilbert was referring to the case $n=2$ in his problems, but we shall assume only $n \geq 2$ for now; the case $n=1$, which is the context in which the subject began (in 1696, arguably, but certainly no later than 1744), has a markedly different character, and will be considered in the final section. Hilbert also took the function $F(x, u, z)$ (the Lagrangian) to be analytic; the problem is regular if $F_{z z}$ is positive definite everywhere. We do not discuss in this article the so-called vector case of the problem in which $u$ is vector-valued (that is, in which there are several unknown functions).

The decade preceding the formulation of Hilbert's problems had been marked by a controversy over the Dirichlet principle, which affirms the equivalence between functions $u$ minimizing the Dirichlet functional $(n=2)$

$$
\int_{\Omega}\left(u_{x}^{2}+u_{y}^{2}\right) d x d y
$$

and solutions $u$ of Laplace's equation $u_{x x}+u_{y y}=0$. As Weierstrass and Hilbert pointed out in response to (notably) Riemann's assertions, the existence of a minimum here (and the very class in which to seek one) is problematic. Hilbert went on to give the first rigorous treatment of the issue in 1904, in a context which succeeded in limiting the class of functions $u$ involved to Lipschitz ones. But it became clear that a more general type of function space was needed, and eventually the work of Levi, Tonelli, Morrey, Sobolev and others, led to the theory of Sobolev spaces, which provides a suitable context in which to assert the existence of a solution to the basic problem.

In the meantime, however, significant progress on Hilbert's regularity question was made:

(1) If $u$ is $C^{3}$, then $u$ is analytic [Bernstein 1904];

(2) If $u$ is $C^{2}$, then $u$ is $C^{3}$ [Lichtenstein 1912];

(3) If $u$ is $C^{1, \alpha}$ (that is, has a gradient which is Hölder continuous of order $\alpha \in(0,1])$, then $u$ is $C^{2}$ [Hopf 1929].

These results lowered the regularity threshold to $C^{1, \alpha}$; once this level of regularity is present in the solution, then it is as regular as the Lagrangian permits: $C^{r}(r \geq 2), C^{\infty}$, or analytic.

Letting $W^{1,1}(\Omega)$ denote the usual Sobolev space, we now consider the following reduced basic problem $(\mathrm{P})$ :

$$
\operatorname{minimize} J(u):=\int_{\Omega} F(D u(x)) d x: u-\phi \in W_{0}^{1,1}(\Omega)
$$


under the following Standing Hypotheses:

(1) $F$ is of class $C^{2}$ and $F_{z z}>0$ everywhere;

(2) $F$ is coercive of order $p>1$ : for certain constants $\sigma>0$ and $\mu$,

$$
F(z) \geq \sigma|z|^{p}+\mu, z \in \mathbb{R}^{n}
$$

(3) $\phi: \mathbb{R}^{n} \rightarrow \mathbb{R}$ is Lipschitz.

It is a standard exercise in the theory of Sobolev spaces to invoke the direct method introduced by Tonelli, in which one exploits the weak sequential compactness of a minimizing sequence and the weak lower semicontinuity of the convex functional $J$, to deduce the existence of a solution $u$ to problem (P) ( $u$ is the unique solution, since $J$ is strictly convex). Note that this is an answer of sorts to Hilbert's 20th problem. The issue now becomes the regularity of the solution $u$, especially since functions in the Sobolev space $W^{1,1}(\Omega)$ are not even continuous necessarily.

The reasons for wanting regularity of the solution $u$ are manifold. For example, continuity of $u$ (or more precisely, of one of its representaives) on the closure $\bar{\Omega}$ of $\Omega$ would mean that the boundary conditions are assumed in the conventional pointwise manner (rather than in the sense of trace). Differentiability of $u$ would mean that $D u$ can be interpreted as the true gradient, and not just the weak distributional derivative. If $u$ is locally Lipschitz in $\Omega$ (or equivalently, Lipschitz on compact subsets of $\Omega$ ), then the Euler equation in weak form can be asserted to hold:

$$
\int_{\Omega} \nabla F(D u(x)) \cdot D \psi(x) d x=0, \psi \in C_{c}(\Omega) .
$$

And finally, in view of the results cited above, local $C^{1, \alpha}$ regularity would imply that the full regularity of the Lagrangian $F$ is inherited by $u$, up to and including analyticity (as in Hilbert's 19th problem).

There are two major 20th century developments on the regularity issue to report, and both were first obtained in the context of the reduced problem $(\mathrm{P})$. We now examine these in turn.

\section{The theorem of De Giorgi}

The Lagrangian $F$ is said to uniformly elliptic provided that for some $\epsilon>0$ we have $F_{z z}(z) \geq \epsilon I$ for all $z \in \mathbb{R}^{n}$. It is said to be almost quadratic if for certain constants $c_{0}, c_{1}, d_{0}>0, d_{1}>0$ we have

$$
c_{0}+d_{0}|z|^{2} \leq F(z) \leq c_{1}+d_{1}|z|^{2}, z \in \mathbb{R}^{n} .
$$


The Dirichlet integrand, which is precisely quadratic, is the canonical example of such a Lagrangian. As we know from harmonic analysis, its minimizers are analytic in $\Omega$. In 1957, De Giorgi? proved the following celebrated result:

Theorem 2.1. Let $F$ be $C^{2}$, uniformly elliptic and almost quadratic. Then the solution $u$ to $(P)$ is locally $C^{1, \alpha}$ in $\Omega$.

Note that the boundary function $\phi$ plays no role here, and that the theorem is strictly one of 'interior regularity'. De Giorgi's proof proceeded by obtaining a linearized Euler equation for $u$, to which a new regularity result on elliptic pde's was then applied to get the required conclusion. This difficult result was introduced in the same article, and other proofs of it were later given by Nash (1958), and by Moser (1960).

The main effect of the theorem, from the point of view of the present discussion, is to reduce the regularity threshold to 'locally Lipschitz'. Let us make this explicit by recording the following simple consequence of the theorem which, surprisingly, is not stated in De Giorgi's article:

Corollary 2.1. Under merely the hypotheses that $F$ is $C^{2}$ and regular, if the solution $u$ to the problem $(P)$ is locally Lipschitz, then it is locally $C^{1, \alpha}$.

This is proved by replacing the original Lagrangian by one which agrees with $F$ on a bounded set containing the values of $D u(x)$ and which is uniformly elliptic and almost quadratic. Then $u$ is still a solution of the problem (by convexity), and De Giorgi's theorem applies to yield the conclusion.

The theorem above has been extended in a variety of ways. Certain evident limits to such extensions, however, as well as possible grounds for pessimism, arise from certain examples due to Giusti and Giaquinta. We refer here to special cases of $(\mathrm{P})$ in which the Lagrangian is uniformly elliptic and satisfies

$$
c_{0}+d_{0}|z|^{2} \leq F(z) \leq c_{1}+d_{1}|z|^{4}, z \in \mathbb{R}^{n},
$$

and yet the solution fails to be continuous in $\Omega$. In such examples, though, the boundary function $\phi$ is itself discontinuous. This motivates the thought that if some regularity properties were imposed on $\phi$, then perhaps this would induce regularity of the solution $u$.

This idea, which harkens back to Hilbert's successful analysis of the Dirichlet principle, is precisely the one that underlies the other significant approach to the regularity issue; we turn to it now. 


\section{Hilbert-Haar theory}

The classical Hilbert-Haar approach, which in contrast to Theorem ?? makes no additional structural assumptions on $F$, requires instead that $\phi$ satisfy the bounded slope condition (BSC) defined below. The ingredients of the theory come from several sources. Hilbert is responsible for the first version of the comparison (or maximum) principle (in the Dirichlet context), which Haar extended to other Lagrangians. Rado introduced the 'three point condition', a forerunner of the BSC below. The idea of applying comparison to a translated solution is due to von Neumann. The BSC was formulated and studied in its present form by Hartman and Nirenberg, while Stampacchia? coined the term BSC and applied it to variational problems.

The bounded slope condition of rank $K$ is the requirement that given any point $\gamma$ on the boundary, there exist two affine functions

$$
y \mapsto\left\langle\zeta_{\gamma}^{-}, y-\gamma\right\rangle+\phi(\gamma), y \mapsto\left\langle\zeta_{\gamma}^{+}, y-\gamma\right\rangle+\phi(\gamma)
$$

agreeing with $\phi$ at $\gamma$ whose 'slopes' satisfy $\left|\zeta_{\gamma}^{-}\right| \leq K,\left|\zeta_{\gamma}^{+}\right| \leq K$ and such that

$$
\left\langle\zeta_{\gamma}^{-}, \gamma^{\prime}-\gamma\right\rangle+\phi(\gamma) \leq \phi\left(\gamma^{\prime}\right) \leq\left\langle\zeta_{\gamma}^{+}, \gamma^{\prime}-\gamma\right\rangle+\phi(\gamma) \quad \forall \gamma^{\prime} \in \Gamma .
$$

Let $\operatorname{Lip}(\Omega)$ denote the class of globally Lipschitz functions on $\Omega$. The Hilbert-Haar theorem (see Chapter 1 of Giusti? ${ }^{\text {? }}$ ) is the following:

Theorem 3.1. Let $\phi$ satisfy the BSC of rank $K$. Then there is a solution $u$ of problem $(P)$ when it is restricted to $\operatorname{Lip}(\Omega)$, and the solution $u$ is Lipschitz on $\Omega$ of rank $K$.

It is possible to show ${ }^{?, ?, ?}$ that the solution of $(\mathrm{P})$ relative to $\operatorname{Lip}(\Omega)$ is in fact the solution relative to $W^{1,1}(\Omega)$, so we now deduce

Corollary 3.1. If $\phi$ satisfies the BSC of rank $K$, then the solution $u$ of problem $(P)$ is Lipschitz on $\Omega$ of rank $K$.

It is clear that Theorems ?? and ??, or rather their corollaries, work in tandem to assert the higher regularity of the solution $u$ to $(\mathrm{P})$, as follows: if $\phi$ satisfies the BSC, then $u$ is locally $C^{1, \alpha}$ and inherits the full regularity of the Lagrangian up to analyticity.

It is natural to ask now how restrictive the BSC is. It is certainly a serious limitation of the allowable boundary conditions on 'flat parts' of $\Gamma$, since it forces $\phi$ to be affine. But the BSC becomes more interesting when $\Omega$ is sufficiently curved. $\Omega$ is said to be uniformly convex if, for some $\epsilon>0$, 
every point $\gamma$ on the boundary admits a hyperplane $H$ through $\gamma$ such that

$$
d_{H}\left(\gamma^{\prime}\right) \geq \epsilon\left|\gamma^{\prime}-\gamma\right|^{2} \quad \forall \gamma^{\prime} \in \Gamma .
$$

Miranda's Theorem ${ }^{?}$ states that when $\Omega$ is uniformly convex, then any $\phi$ of class $C^{2}$ satisfies the BSC. Later, Hartman? showed that when $\Omega$ is uniformly convex and $\Gamma$ is $C^{1,1}$, then $\phi$ satisfies the BSC if and only if $\phi$ is itself $C^{1,1}$. We can say therefore that as a hypothesis, the BSC essentially restricts the boundary data to be smooth. When $\phi$ is not affine, the BSC also forces $\Omega$ to be convex, a hypothesis that we will add to our Standing Hypotheses as we turn now to some new results that center around a weakening of the BSC.

\section{New boundary hypotheses}

We now turn our attention to the present, or at least the very recent past. We assume throughout this section that $\Omega$ is convex. Clarke? has introduced a new hypothesis on $\phi$, the lower bounded slope condition (lower BSC) of rank $K$ : given any point $\gamma$ on the boundary, there exists an affine function $y \mapsto\left\langle\zeta_{\gamma}, y-\gamma\right\rangle+\phi(\gamma)$ with $\left|\zeta_{\gamma}\right| \leq K$ such that

$$
\left\langle\zeta_{\gamma}, \gamma^{\prime}-\gamma\right\rangle+\phi(\gamma) \leq \phi\left(\gamma^{\prime}\right) \quad \forall \gamma^{\prime} \in \Gamma .
$$

Being one-sided, the lower BSC naturally admits a counterpart: an upper BSC that is satisfied by $\phi$ exactly when $-\phi$ satisfies the lower BSC.

\subsection{Interior regularity}

The significance of the 'partial' BSC hypothesis stems from the following result:?

Theorem 4.1. If $\phi$ satisfies the lower bounded slope condition, then the solution $u$ of $(P)$ is locally Lipschitz in $\Omega$. In fact, there is a constant $\bar{K}$ with the property that for any subdomain $\Omega^{\prime}$ of distance $\delta>0$ from $\Gamma$, we have

$$
|u(x)-u(y)| \leq(\bar{K} / \delta)|x-y| \quad \forall x, y \in \Omega^{\prime} .
$$

Thus the one-sided BSC gives the crucial regularity property: $u$ is locally Lipschitz in $\Omega$. This allows us to assert that $u$ is a weak solution of the Euler equation, in the absence of any restrictive growth conditions on $F$, and of course allows the application of Theorem ?? to deduce higher-order 
regularity. Informally, it appears that in return for 'half the BSC hypothesis', we obtain considerably more than half the conclusion. Of course the principal thing that has been sacrificed is the continuity of $u$ up to the boundary, but as we shall see below, this can be recovered under a variety of additional hypotheses.

As in the case of the BSC, it behooves us to examine the conditions under which the one-sided BSC can be asserted to hold. In this new context, the property that $\Omega$ be curved has less importance than before; flat parts of the boundary do not force $\phi$ to be affine. Nonetheless, curvature can still serve a purpose: Bousquet? has shown that when $\Omega$ is uniformly convex, then $\phi$ satisfies the lower (upper) BSC if and only if it is the restriction to $\Gamma$ of a function which is semiconvex (semiconcave), a familiar and useful property in pde's (see for example Ref. ?). In the uniformly convex case, therefore, Theorem ?? extends Hilbert-Haar theory to boundary data that is semiconvex or semiconcave rather than $C^{2}$ (or $C^{1,1}$ ).

We remark that the proofs of Theorems ?? and ?? have something in common: both of them construct a new minimizer from $u$ itself with which to compare $u$. In the classical case, this is done by translation. The principal new idea in the proof of Theorem ?? is to construct a new minimizer through dilation rather than translation.

\subsection{Continuity at the boundary}

In a variety of situations, it turns out that the lower or upper BSC does imply continuity at the boundary, and even a global Hölder condition in some cases. A counterexample due to Bousquet and Cannarsa (in the Dirichlet context) shows, however, that the gradient of $u$ can become unbounded.

Theorem 4.2. Suppose that in addition to the hypotheses of Theorem ??, one of the following holds:

(a) $\Gamma$ is a polyhedron, or

(b) $\Gamma$ is $C^{1,1}$ and $p>(n+1) / 2$, or

(c) $\Omega$ is uniformly convex.

Then $u$ is continuous on $\bar{\Omega}$. In cases (a) and (b), u satisfies a Hölder condition on $\bar{\Omega}$.

We remark that we know of no example in which (under the hypotheses of Theorem ??) the solution fails to be continuous on $\bar{\Omega}$. Indeed, we know of no example in which $\phi$ is Lipschitz and $u$ fails to be continuous. 


\subsection{More general Lagrangians}

It turns out to be challenging to extend the new results given above to Lagrangians depending on $x$ and $u$ as well as $D u$, principally because the comparison principle does not hold in that case. However, using the technique of barrier functions, Bousquet and Clarke? have obtained a result for Lagrangians of the form $F(D u)+G(x, u)$; we describe it now. We assume that $F$ is uniformly elliptic, that $G(x, u)$ is measurable in $x$ and differentiable in $u$, and that for every bounded interval $U$ in $\mathbb{R}$ there is a constant $L_{U}$ such that for almost all $x \in \Omega$,

$$
\left|G(x, u)-G\left(x, u^{\prime}\right)\right| \leq L_{U}\left|u-u^{\prime}\right| \forall u, u^{\prime} \in U .
$$

We also postulate that for some bounded function $b$, the integral $\int_{\Omega} G(x, b(x)) d x$ is well-defined and finite.

Theorem 4.3. Under these hypotheses, and when $\phi$ satisfies the Lower or Upper BSC, any bounded solution u of the basic problem is locally Lipschitz in $\Omega$.

We remark in connection with this result that it is possible to formulate additional hypotheses on the data which imply a priori that any solution to the basic problem is bounded, and that additional structural hypotheses lead as before to continuity at the boundary.

\section{The one-dimensional case}

Finally, let us address the beginning of the subject: the one-dimensional case, for which there is always a different notation. The basic problem $(\mathrm{P})$ now corresponds to the minimization of the functional

$$
J(x):=\int_{a}^{b} F\left(t, x(t), x^{\prime}(t)\right) d t
$$

over the functions $x:[a, b] \rightarrow \mathbb{R}^{N}$ in some given class $X$, and subject to prescribed endpoint conditions: $x(a)=A, x(b)=B$. Note that we now allow $N>1$, the case of several unknown functions; the generic name for the variables becomes $(t, x, v)$ rather than $(x, u, z)$.

For Euler and his contemporaries, all functions were smooth, so the issue of the regularity of the solutions did not arise; implicitly, $X$ was a space of very smooth functions. On a more rigorous level, when the degree of smoothness becomes a consideration, we can deduce that if $x$ is $C^{1}$ to start with, and if $F$ is regular (which continues to mean $F_{v v}>0$ ) and at 
least $C^{2}$, then $x$ inherits the full degree of regularity of $F$, up to analyticity. This is known as the Hilbert-Weierstrass theorem (circa 1890), and is a consequence of the Euler equation together with the implicit function theorem. By the 19th century, however, the possible nonsmoothness of solutions began to be recognized as an important point, in view of such concrete evidence as nonsmooth soap (minimal) surfaces.

Results were obtained for the class PWS of piecewise smooth functions, notably. An important breakthrough was duBois-Reymond's proof of the integral form of the Euler equation: if $x$ solves (P) relative to PWS, then there exists a piecewise smooth function $p$ such that

$$
\left(p^{\prime}(t), p(t)\right)=\nabla F\left(t, x(t), x^{\prime}(t)\right)
$$

at all non-corner points; here, $\nabla F$ refers to the gradient in the $(x, v)$ variables. This condition subsumes the earlier Erdmann condition, and like it, can sometimes be used to deduce the smoothness of $x$ (in this setting, the absence of corners). Note however that $x$ has to be assumed piecewise smooth a priori. This is unfortunate, since, although suitable necessary conditions can be asserted in PWS, the class of piecewise smooth functions is of no help in regard to the existence issue.

It was Tonelli's major contribution to show that existence theory can be developed successfully in the class AC of absolutely continuous functions. But within AC, the ability to derive the Euler equation fails in general, so our steps forward (on existence) are accompanied by one step back (on the necessary conditions). A way out of this quandary is to find reasonable supplementary hypotheses on the Lagrangian which will imply that $x$ is Lipschitz. The reason for this is that all the classical results for the class PWS carry over to this class (now that Lebesgue has given us his integral). In other words, just as in the multi-dimensional case treated in the previous sections, the regularity threshold is situated at Lipschitz continuity of the solution.

Of course, in the one-dimensional case there is little help to be found from examining the boundary conditions (as in the Hilbert-Haar theory). In fact, the methodology used to obtain regularity theorems has been overwhelmingly based upon analyzing the necessary conditions. This is in stark contrast to the multi-dimensional case, where the necessary conditions don't seem to yield very much directly. We conclude therefore that our best hope to extend regularity theory lies in the possibility of deriving stronger necessary conditions in more general circumstances.

We proceed to report on just such a recent development. For this pur- 
pose, we examine a Lagrangian $F(t, x, v)$ which is merely measurable in $t$ and $(x, v)$ (see ${ }^{?}$ for the precise meaning of this) and lower semicontinuous in $(x, v)$. No hypotheses of smoothness or convexity are made. Instead, we assume the following generalized Tonelli-Morrey condition [GTM]: for every bounded subset $S$ of $\mathbb{R}^{n}$ there exist a constant $c$ and a summable function $d$ such that for almost every $t$, for every $(x, v) \in S \times \mathbb{R}^{n}$, for every $(\zeta, \psi) \in \partial_{P} F(t, x, v)$, one has

$$
\frac{|\zeta|}{1+|\psi|} \leq c\{|F(t, x, v)|+|v|\}+d(t) .
$$

Here, $\partial_{P} F$ refers to the proximal subgradient of $F$ with respect to the $(x, v)$ variables, a basic construct in nonsmooth analysis.

When $F$ is $C^{2}$ (or somewhat less), the growth condition of [GTM] is equivalent to

$$
\left|D_{x} F\right| \leq c_{1}\left\{|F|+|v|+\left|D_{v} F\right|\right\}+d_{1}(t)+\left\{c_{2}(|F|+|v|)+d_{2}(t)\right\}\left|D_{v} F\right| .
$$

The special case $c_{2}=d_{2}=0$ corresponds to a class of Lagrangians that has been considered by Clarke and Vinter? in connection with regularity. In this case, and when in addition the term involving $\left|D_{v} F\right|$ is placed on the left side of the inequality, as follows:

$$
\left|D_{x} F\right|+\left|D_{v} F\right| \leq c_{1}\{|F|+|v|\}+d_{1}(t),
$$

(thereby making the condition a more stringent hypothesis), we obtain a growth condition first postulated by Tonelli in order to be able to derive the necessary conditions in the class AC.

In the much more general setting now being considered, the new [GTM] still has the same effect: it allows one to deduce necessary conditions that must be satisfied by any solution to $(\mathrm{P})$. These conditions have the nature of the classical ones, but expressed in such a way as to take account of the nonsmoothness of $F$. Here is just one such result taken from Clarke:?

Theorem 5.1. Let $x$ be a solution to $(P)$ relative to the class $\mathrm{AC}$, where $F$ satisfies the generalized Tonelli-Morrey growth condition [GTM]. Then there exists an arc $p$ satisfying the Euler inclusion

$$
p^{\prime}(t) \in \operatorname{co}\left\{\omega:(\omega, p(t)) \in \partial_{L} F\left(t, x(t), x^{\prime}(t)\right)\right\} \text { a.e., }
$$

and the Weierstrass condition: for almost all $t \in[a, b]$ we have

$$
F(t, x(t), v)-F\left(t, x(t), x^{\prime}(t)\right) \geq\left\langle p(t), v-x^{\prime}(t)\right\rangle \quad \forall v \in \mathbb{R}^{n} .
$$


The Euler inclusion in this statement involves the limiting subdifferential $\partial_{L} F$; it reduces to the integral form of the Euler equation when $F$ is smooth. The Weierstrass condition is the familiar one of the classical theory.

This theorem admits a more general form in which the cost depends on the endpoint values of the arc $x$, which need be only a local minimum in a specified sense. Beyond this, and most significantly, the theorem can be stated for extended-valued Lagrangians $F$, so that problems in optimal control are subsumed by it. But these are chapters in a different story, so let us proceed instead with our quest for regularity consequences.

We say that the Lagrangian $F$ is coercive if for any bounded subset $S$ of $\mathbb{R}^{n}$ there exists a function $\theta:[0, \infty) \rightarrow \mathbb{R}$ satisfying

$$
\lim _{r \rightarrow+\infty} \frac{\theta(r)}{r}=+\infty
$$

and such that

$$
F(t, x, v) \geq \theta(|v|) \quad \forall(t, x, v) \in[a, b] \times S \times \mathbb{R}^{n} .
$$

We remark that coercivity is a familiar ingredient in the theory of existence of solutions. The symbiosis between necessary conditions and regularity is well illustrated by the following new result.

Corollary 5.1. If $x$ solves $(P)$ relative to the class $\mathrm{AC}$, where $F$ is coercive, bounded above on bounded sets, and satisfies the generalized Tonelli-Morrey growth condition $[\mathrm{GTM}]$, then $x$ is Lipschitz on $[a, b]$.

Proof. In view of Theorem ??, we know that an arc $p$ exists which satisfies the Weierstrass condition. Let $M$ be an upper bound on

$$
F\left(t, x(t), \frac{x^{\prime}(t)}{1+\left|x^{\prime}(t)\right|}\right)
$$

for $t \in[a, b]$, and let $\theta$ be a coercivity function for $F$ when the variable $x$ is restricted to the bounded set consisting of the values of $x(t)$ on $[a, b]$. Then, taking $v:=x^{\prime}(t) /\left(1+\left|x^{\prime}(t)\right|\right)$ in the Weierstrass inequality leads to (almost everywhere)

$$
\theta\left(\left|x^{\prime}(t)\right|\right) \leq F\left(t, x(t), x^{\prime}(t)\right) \leq M+|p(t)|\left|x^{\prime}(t)\right| .
$$

Since $|p(t)|$ is bounded and $\lim _{r \rightarrow \infty} \theta(r) / r=+\infty$, it follows from this inequality that $x^{\prime}(t)$ is essentially bounded on $[a, b]$. 
This corollary even extends (relative to previous results) the class of smooth Lagrangians for which the necessary conditions can be asserted and regularity inferred. A simple example of this is provided (for $N=1$ ) by

$$
F(t, x, v)=\exp \left\{\left(1+x^{2}+t^{2}\right) v^{2}\right\} .
$$

This Lagrangian satisfies the hypotheses of the classical Tonelli existence theorem as well as those of the corollary. Thus a solution to (P) over AC exists, and any solution $x$ is Lipschitz. Because $F$ is strictly convex in $v$, it then follows? that $x$ is $C^{1}$, and finally we derive all the higher regularity of $x$ from the Hilbert-Weierstrass theorem.

There are other structural hypotheses yielding regularity results that serve to highlight the extremely general Lagrangians that can be treated in the one-dimensional case, as compared to the very special structure that seems to be required in the multi-dimensional case. We end the discussion with one further example, ${ }^{\text {? }}$ which asserts the regularity of the solution for one-dimensional problems having autonomous Lagrangians ( $F$ is called autonomous when it has no explicit dependence on the variable $t$ ).

Theorem 5.2. Let $x$ solve $(P)$ relative to $\mathrm{AC}$, where the Lagrangian $F$ is coercive, bounded above on bounded sets, and autonomous. Then $x$ is Lipschitz on $[a, b]$.

This result (among others that we do not touch upon here) was first proved by Clarke and Vinter? under the added requirement that $F$ be locally Lipschitz and convex in $v$. A more direct and simplified proof in that setting can be given.?

The literature on the venerable subject of regularity in the calculus of variations, and on the inextricably linked issues of existence and necessary conditions, is huge and still growing. We make no attempt here to be complete, but rather we refer the interested reader to the classic books of Giaquinta, Giusti and Morrey, and to the representative (but not exhaustive) more recent references appearing below, in which detailed bibliographic information appears.

\section{References}

1. P. Bousquet, J. Convex Analysis 14, 119 (2007).

2. P. Bousquet and F. Clarke, J. Differential Equations 243, 489 (2007).

3. G. Buttazzo and M. Belloni, A survey on old and recent results about the gap phenomenon, in Recent Developments in Well-Posed Variational Problems, eds. R. Lucchetti and J. Revalski (Kluwer, Dordrecht, 1995) pp. 1-27. 
4. G. Buttazzo, M. Giaquinta and S. Hildebrandt, One-dimensional Variational Problems, Oxford Lecture Series in Mathematics and its Applications, Vol. 15 (Clarendon Press, 1998).

5. P. Cannarsa and C. Sinestrari, Semiconcave Functions, Hamilton-Jacobi Equations, and Optimal Control (Birkhäuser, Boston, 2004).

6. A. Cellina, SIAM J. Control Optim. 40, 1270 (2001).

7. F. H. Clarke, Regularity, existence and necessary conditions for the basic problem in the calculus of variations, in Contributions to the Modern Calculus of Variations, ed. L. Cesari (Longman, London, 1987).

8. F. H. Clarke, Trans. Amer. Math. Soc. 336, 655 (1993).

9. F. Clarke, Ann. Scuola Norm. Sup. Pisa Cl. Sci. (5) 4, 511 (2005).

10. F. Clarke, Necessary Conditions in Dynamic Optimization, Memoirs of the Amer. Math. Soc., No. 816, Vol. 173, 2005.

11. F. Clarke, Ergodic Theory Dynam. Systems 27, 1 (2007).

12. F. H. Clarke and R. B. Vinter, J. Differential Equations 59, 336 (1985).

13. F. H. Clarke and R. B. Vinter, Trans. Amer. Math. Soc. 289, 73 (1985).

14. R. De Arcangelis, Ann. Univ. Ferrara 35, 135 (1989).

15. E. De Giorgi, Mem. Accad. Sci. Torino 3, 25 (1957).

16. E. Giusti, Direct Methods in the Calculus of Variations (World Scientific, Singapore, 2003).

17. P. Hartman, Pacific J. Math. 18, 495 (1966).

18. C. Mariconda and G. Treu, Proc. Amer. Math. Soc. 130, 395 (2001).

19. C. Mariconda and G. Treu, J. Optim. Theory Appl. 112, 167 (2002).

20. M. Miranda, Ann. Scuola Norm. Sup. Pisa Cl. Sci. (3) 19, 233 (1965).

21. G. Stampacchia, Comm. Pure Appl. Math. 16, 383 (1963). 